\title{
Gravitational wave generation in power-law inflationary models
}

\author{
Paulo M. Sá \\ Centro Multidisciplinar de Astrofísica - CENTRA and Departamento de Física, \\ FCT, Universidade do Algarve, Campus de Gambelas, 8005-139 Faro, Portugal \\ Alfredo B. Henriques \\ Centro Multidisciplinar de Astrofísica - CENTRA and Departamento de Física, \\ Instituto Superior Técnico, UTL, Av. Rovisco Pais, 1049-001 Lisboa, Portugal
}

(Dated: June 5, 2008)

\begin{abstract}
We investigate the generation of gravitational waves in power-law inflationary models. The energy spectrum of the gravitational waves is calculated using the method of continuous Bogoliubov coefficients. We show that, by looking at the interval of frequencies between $10^{-5}$ and $10^{5} \mathrm{~Hz}$ and also at the $\mathrm{GHz}$ range, important information can be obtained, both about the inflationary period itself and about the thermalization regime between the end of inflation and the beginning of the radiation-dominated era. We thus deem the development of gravitational wave detectors, covering the $\mathrm{MHz} / \mathrm{GHz}$ range of frequencies, to be an important task for the future.

PACS numbers: 04.30.Db, 98.70.Vc, 98.80.Cq
\end{abstract}

\section{INTRODUCTION}

Gravitational waves have the potential of providing us with a unique telescope and a unique source of information about the very early universe.

Since the first calculation of the full spectrum of stochastic gravitational waves has been performed by Allen [1], continuing and completing work by other authors, including Grishchuk [2] and Starobinsky [3], Rubakov, Sazhin and Veryaskin [4], as well as by Fabbri and Pollock [5], Abbott and Wise [6], and Abbott and Harari 7], a lot of research has been done in this direction, particulary into exploring the potentialities of different inflationary models. Although the values obtained for the relative energy spectrum of the cosmological gravitational waves seem, at present, to be beyond the capabilities of the first generations of gravitational-wave detectors, this is a situation that is bound to be changed, given the effort that is being put into the planning of future detectors with considerably improved sensitivities like, for instances, NASA's Big Bang Observatory (BBO) [8] and the japanese Deci-hertz Interferometer Gravitational Wave Observatory (DECIGO) [9].

An interesting question to ask is what can we learn about the physics of the very early universe, from the reading of the spectrum, assuming an ideal detector. In this article, we address this question with the help of a popular class of models, namely, power-law inflation. We shall show that, by looking at the interval of frequencies between $10^{-5}$ and $10^{5} \mathrm{~Hz}$, and also at the $\mathrm{GHz}$ range, important information can be obtained, about both the inflationary period and the thermalization regime. Concerning the first range of frequencies, between $10^{-5}$ and $10^{5} \mathrm{~Hz}$, important work, along the lines of our paper, had already been done [10], albeit by a different method, with similar conclusions to our own. Most of the developments that have taken place in gravitational wave detectors cover the first range of frequencies, but work is also going on to study the possibility of constructing detectors covering the $\mathrm{MHz}$ and $\mathrm{GHz}$ range of frequencies [11]. We deem these last developments to be very important, given the amount of information that can thereby be obtained, as we show with the help of a simple model for the transition between the inflationary and radiationdominated eras.

In this paper, we consider those gravitational waves generated by the usual mechanism of quantum fluctuations of the vacuum. In the $\mathrm{MHz} / \mathrm{GHz}$ region another independent mechanism is possible, when the end of inflation is followed by a period of parametric resonance [12], in which case the gravitational waves are directly sourced by the large inhomogeneities in the matter distribution occurring during this period. In this paper we shall not address this important case, which has been investigated elsewhere [13, 14], using both mechanisms.

Throughout, gravitational wave production will be calculated using the method of continuously evolving Bogoliubov coefficients. This method, applied to the production of particles in an expanding universe, was first investigated by Parker [15]. In the case of gravitational waves, the differential equations determining the continuous Bogoliubov coefficients were derived afterwards in a different, geometrical, way [16]. The method of continuous Bogoliubov coefficients has advantages over the frequently used sudden transition approximation. Associated with the sudden transition there is always an overproduction of gravitons of large frequencies, requiring an explicit cut-off for frequencies above the rate of expansion of the universe [1]. This cut-off is obtained in a natural way by the use of continuous Bogoliubov coefficients [17]. This is also a very practical method to calculate the full spectrum, from the very low frequencies corresponding to the present cosmological horizon, till those large, $\mathrm{GHz}$, frequencies associated with the transition between inflation and the radiation-dominated universe.

This paper is organized as follows. In Sec. II we in- 
troduce the equations defining the power-law inflationary model. The parameters of the inflationary potential are constrained using recent measurements from the cosmic microwave background and large-scale structure. In Sec. III reheating is incorporated in our model of evolution through an elementary decay mechanism of the inflationary scalar field into a relativistic radiation fluid. Despite its simplicity, our reheating potential may contain relevant features of more realistic potentials. In Sec. IV we present the differential equations to determine the continuous Bogoliubov coefficients and address the relevant issue of the initial conditions to be used in the numerical integration of these equations. In Sec. $\mathrm{V}$ we describe our numerical simulations and present several gravitational-wave spectra, obtained for different values of the parameters of our model. The paper ends with the Conclusions.

\section{POWER-LAW INFLATION}

Let us assume that the evolution of the universe during the inflationary period is dominated by an homogeneous scalar field $\phi$ with a potential

$$
V(\phi)=V_{0} e^{-\lambda \phi},
$$

where $V_{0}$ and $\lambda$ are positive constants. For a spatially flat Friedmann-Robertson-Walker metric,

$$
d s^{2}=a^{2}(\eta)\left(-d \eta^{2}+d \mathbf{x}^{2}\right),
$$

the Einstein equations for $\phi(\eta)$ and $a(\eta)$ are

$$
\begin{aligned}
& \left(\frac{a^{\prime}}{a}\right)^{2}=\frac{8 \pi}{3 m_{\mathrm{P}}^{2}} a^{2}\left[\frac{\phi^{\prime 2}}{2 a^{2}}+V(\phi)\right], \\
& \phi^{\prime \prime}+2 \frac{a^{\prime}}{a} \phi^{\prime}+a^{2} \frac{d V}{d \phi}=0, \\
& \frac{a^{\prime \prime}}{a}=-\frac{4 \pi}{3 m_{\mathrm{P}}^{2}} a^{2}\left[\frac{\phi^{2}}{a^{2}}-4 V(\phi)\right],
\end{aligned}
$$

where a prime denotes a derivative with respect to conformal time $\eta$ and $m_{\mathrm{P}}=1 / \sqrt{G}=1.22 \times 10^{19} \mathrm{GeV}$ is the Planck mass.

The above set of differential equations admits the exact solution [18]

$$
\begin{aligned}
& a(\eta)=a_{1}\left(\eta_{1}-\eta\right)^{p}, \\
& \phi(\eta)=\frac{1}{\lambda} \ln \left\{\frac{8 \pi}{m_{\mathrm{P}}^{2}} \frac{a_{1}^{2} V_{0}}{p(2 p-1)}\left(\eta_{1}-\eta\right)^{2(p+1)}\right\},
\end{aligned}
$$

where $p<-1, a_{1}$ and $\eta_{1}$ are arbitrary constants, and

$$
\lambda=4 \sqrt{\pi \frac{p+1}{p}} m_{\mathrm{P}}^{-1} .
$$

Inflationary cosmology predicts a nearly scaleinvariant power spectrum of density perturbations [19].
Within the slow-roll approximation, the power spectrum is given by [20]

$$
P_{s}(k)=P_{s}\left(k_{c}\right)\left(\frac{k}{k_{c}}\right)^{1-n_{s}+\left(\alpha_{s} / 2\right) \ln \left(k / k_{c}\right)},
$$

where the scalar power-spectrum amplitude $P_{s}\left(k_{c}\right)$ and the spectral parameters $n_{s}\left(k_{c}\right)$ and $\alpha_{s}\left(k_{c}\right)$ are evaluated at some pivot wave number $k_{c}$. In this paper, we take for these quantities the values $P_{s}\left(k_{c}\right)=(2.45 \pm 0.23) \times 10^{-9}$, $n_{s}\left(k_{c}\right)=1.0 \pm 0.1$ and $\left|\alpha_{s}\left(k_{c}\right)\right|<0.04$ at the pivot wave number $k_{c}=0.05 \mathrm{Mpc}^{-1}$, corresponding to distance scales of the cosmic microwave background (CMB) and large-scale structure (LSS) [20].

The spectral parameters $n_{s}$ and $\alpha_{s}$ can be defined in terms of the usual slow-roll parameters $\epsilon, \bar{\eta}$ and $\xi$, namely, $n_{s}=1-6 \epsilon+2 \bar{\eta}[21]$ and $\alpha_{s}=16 \epsilon \bar{\eta}-24 \epsilon^{2}-2 \xi[22]$. For the exponential potential (1), the slow-roll parameters are $\epsilon=(p+1) / p, \bar{\eta}=2(p+1) / p$ and $\xi=4(p+1)^{2} / p^{2}$, yielding $n_{s}=-(p+2) / p$ and $\alpha_{s}=0$.

Taking into account the above relation between $n_{s}$ and $p$ and the CMB/LSS constraint on $n_{s}$, we conclude that $p \geqslant-2 / 1.9 \approx-1.053$. Despite the fact that $p$ takes values within a very narrow window, $-1.053 \leqslant p<-1$, it has quite a dramatic effect on the energy spectrum of the gravitational waves, as will be shown in Sec. V.

Within the slow-roll approximation, the power spectrum (9) can be written as

$$
P_{s}(k)=\left.\frac{128 \pi}{3 m_{\mathrm{P}}^{6}} \frac{V^{3}}{(d V / d \phi)^{2}}\right|_{\phi=\phi_{c}}=\frac{8 V_{0} e^{-\lambda \phi_{c}}}{3 m_{\mathrm{P}}^{4}} \frac{p}{p+1},
$$

where $\phi_{c} \equiv \phi\left(\eta_{c}\right)$ is the value of the scalar field at the moment when the scale $k_{c}$ exits the Hubble horizon during the inflationary period. Within our model, $\phi_{c}$ can be chosen freely. Let us express it in terms of $\phi_{i} \equiv \phi\left(\eta_{i}\right)$, the value of the scalar field at the time reheating begins. Taking into account that the number of e-foldings of expansion between $\phi_{c}$ and $\phi_{i}$ is given by

$$
N_{c}=\frac{8 \pi}{m_{\mathrm{P}}^{2}} \int_{\phi_{i}}^{\phi_{c}} \frac{V}{d V / d \phi} d \phi,
$$

we obtain

$$
\phi_{c}=\phi_{i}-N_{c} \sqrt{\frac{p+1}{4 \pi p}} m_{\mathrm{P}} .
$$

Inserting this expression into Eq. (10), and using Eq. (9), we obtain a constraint on the inflationary scale $V_{0}$, namely,

$$
\begin{aligned}
V_{0}= & \frac{3(p+1)}{8 p} P_{s}\left(k_{c}\right) \\
& \times \exp \left\{4 \sqrt{\pi \frac{p+1}{p}} \frac{\phi_{i}}{m_{\mathrm{P}}}-2 N_{c} \frac{p+1}{p}\right\} m_{\mathrm{P}}^{4},
\end{aligned}
$$

where $47 \lesssim N_{c} \lesssim 62[20]$ and, as already mentioned above, $\phi_{i}$ is a free parameter, marking the beginning of 


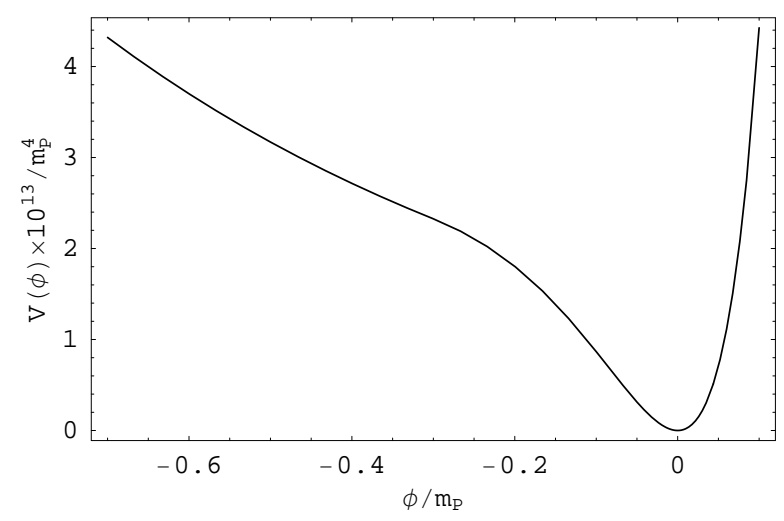

FIG. 1: Potential $V(\phi)$ for $p=-1.05, \phi_{i}=-0.3 m_{\mathrm{P}}$ and $n=2$, implying $\lambda=1.547 m_{\mathrm{P}}^{-1}, V_{0}=1.461 \times 10^{-13} m_{\mathrm{P}}^{4}, U_{0}=$ $2.785 \times 10^{-13} m_{\mathrm{P}}^{4}$ and $\nu=8.152 m_{\mathrm{P}}^{-1}$.

the reheating process. In the numerical simulations, described in detail in Sec. V] we will use $\phi_{i}=-0.3 m_{\mathrm{P}}$, $P_{s}\left(k_{c}\right)=2.45 \times 10^{-9}$ and $N_{c}=55$, implying that $V_{0}^{1 / 4} \leqslant 1.5 \times 10^{16} \mathrm{GeV}$ for $-1.053 \leqslant p<-1$.

\section{REHEATING}

In order to incorporate reheating in our model of evolution, we assume that the potential of the scalar field is no longer given by Eq. (11), but rather by

$$
V(\phi)= \begin{cases}V_{0} e^{-\lambda \phi}, & \phi \leqslant \phi_{i}, \\ U_{0}\left(e^{\nu \phi}-1\right)^{n}, & \phi \geqslant \phi_{i},\end{cases}
$$

where $n$ is a even number and $U_{0}$ and $\nu$ are chosen such that the potential and its first derivative are continuous at $\phi=\phi_{i}$ (see Fig. 1).

This is clearly a toy potential. However, it may contain relevant features of more realistic potentials, arising from extensions of the standard model; these are likely to involve potentials defined by power-series expansions of scalar fields, or by exponentials [23]. An important example of the last type, may be seen in the application of the Salam-Sezgin six-dimensional supergravity model [24] to cosmology 25]. Our potential is similar to the one derived within the Salam-Sezgin model, if in Eq. (14) we put $n=2$.

Initially, the scalar field $\phi$ has a large negative value. A period of pure power-law inflation takes place until $\phi$ rolls down to values of the order of $\phi_{i}$. After that, the scalar field rapidly approaches the minimum of the potential, where $V \approx U_{0} \nu^{n} \phi^{n}$, beginning to oscillate around it. The energy of the scalar field is then transferred to a relativistic radiation fluid and reheating takes place. The decay rate of the scalar field into the radiation fluid is governed by a phenomenological parameter, $\Gamma_{\phi}$, which we choose to be of the order of $10^{-7} m_{\mathrm{P}}-10^{-8} m_{\mathrm{P}}$.

During the period of transition between power-law inflation and the radiation-dominated era, the evolution of the universe is described by the set of differential equations

$$
\begin{aligned}
& \left(\frac{a^{\prime}}{a}\right)^{2}=\frac{8 \pi}{3 m_{\mathrm{P}}^{2}} a^{2}\left[\frac{\phi^{2}}{2 a^{2}}+U_{0}\left(e^{\nu \phi}-1\right)^{n}+\rho_{\mathrm{rad}}\right] \\
& \frac{a^{\prime \prime}}{a}=-\frac{4 \pi}{3 m_{\mathrm{P}}^{2}} a^{2}\left[\frac{\phi^{\prime 2}}{a^{2}}-4 U_{0}\left(e^{\nu \phi}-1\right)^{n}\right] \\
& \phi^{\prime \prime}+2 \frac{a^{\prime}}{a} \phi^{\prime}+a^{2} n \nu U_{0}\left(e^{\nu \phi}-1\right)^{n-1} e^{\nu \phi}=-\Gamma_{\phi} a \phi^{\prime}, \\
& \rho_{\mathrm{rad}}^{\prime}+4 \frac{a^{\prime}}{a} \rho_{\mathrm{rad}}=\Gamma_{\phi} \frac{\phi^{\prime 2}}{a}
\end{aligned}
$$

where $\rho_{\text {rad }}$ is the energy density of the relativistic radiation fluid.

The transition period ends when the energy density of the scalar field, $\rho_{\phi}=\phi^{\prime 2} /\left(2 a^{2}\right)+U_{0}\left(e^{\nu \phi}-1\right)^{n}$, becomes much smaller than the energy density of radiation, $\rho_{\text {rad }}$. After that, we neglect the scalar field and the evolution of the universe till the present time is described by the set of differential equations

$$
\begin{aligned}
\frac{a^{\prime \prime}}{a}= & \frac{4 \pi G}{3} a^{2}\left[\rho_{\mathrm{mat}, 0}\left(\frac{a_{0}}{a}\right)^{3}\right. \\
& \left.+(3 w+1) \rho_{\mathrm{de}, 0}\left(\frac{a_{0}}{a}\right)^{3(1-w)}\right], \\
\left(\frac{a^{\prime}}{a}\right)^{2}= & \frac{8 \pi G}{3} a^{2}\left[\rho_{\mathrm{rad}, 0}\left(\frac{a_{0}}{a}\right)^{4}+\rho_{\mathrm{mat}, 0}\left(\frac{a_{0}}{a}\right)^{3}\right. \\
& \left.+\rho_{\mathrm{de}, 0}\left(\frac{a_{0}}{a}\right)^{3(1-w)}\right],
\end{aligned}
$$

where $w=0.78$ and the density of radiation, matter and dark energy at the present time $\eta_{0}$ are, respectively, $\rho_{\text {rad }, 0}=4.6 \times 10^{-31} \mathrm{~kg} / \mathrm{m}^{3}, \rho_{\text {mat }, 0}=2.6 \times$ $10^{-27} \mathrm{~kg} / \mathrm{m}^{3}$ and $\rho_{\mathrm{de}, 0}=6.9 \times 10^{-27} \mathrm{~kg} / \mathrm{m}^{3}$, yielding $H\left(\eta_{0}\right)=71 \mathrm{~km} \mathrm{~s}^{-1} \mathrm{Mpc}^{-1}$.

\section{GRAVITATIONAL-WAVE SPECTRUM}

The dimensionless relative logarithmic energy spectrum of the gravitational waves at the present time $\eta_{0}$ is defined by

$$
\begin{aligned}
\Omega_{\mathrm{GW}}\left(\eta_{0}\right) & =\frac{1}{\rho_{\mathrm{crit}}\left(\eta_{0}\right)} \frac{d \rho_{\mathrm{GW}}}{d \ln \omega}\left(\eta_{0}\right) \\
& =\frac{8 \hbar G}{3 \pi c^{5} H^{2}\left(\eta_{0}\right)} \omega^{4}\left(\eta_{0}\right) \beta^{2}\left(\eta_{0}\right),
\end{aligned}
$$

where $\rho_{\text {crit }}$ is the critical density of the universe, $\rho_{\mathrm{GW}}$ and $\omega$ are the energy density and angular frequency of the gravitational waves, respectively, $H$ is the Hubble parameter and $\beta$ is a Bogoliubov coefficient, such that $|\beta|^{2}=\left\langle N_{k}(\eta)\right\rangle$ gives the number of gravitons.

In order to calculate the amount of gravitons produced during the evolution of the universe we use Parker's method of continuous Bogoliubov coefficients [15, 16], 
according to which the Bogoliubov coefficients $\alpha(\eta)$ and $\beta(\eta)$, defined as continuous functions of time, are determined by the set of differential equations

$$
\begin{aligned}
\alpha^{\prime} & =\frac{i}{2 k}\left[\alpha+\beta e^{2 i k\left(\eta-\eta_{i}\right)}\right] \frac{a^{\prime \prime}}{a}, \\
\beta^{\prime} & =-\frac{i}{2 k}\left[\beta+\alpha e^{-2 i k\left(\eta-\eta_{i}\right)}\right] \frac{a^{\prime \prime}}{a},
\end{aligned}
$$

where $k=2 \pi a(\eta) / \lambda(\eta)=a(\eta) \omega(\eta)$ is the co-moving wave number. This method has been used by us in previous works to investigate the generation of gravitational waves in different cosmological models [14, 17, 26].

Upon the redefinition

$$
\begin{aligned}
& \alpha=\frac{1}{2}(X+Y) e^{i k\left(\eta-\eta_{i}\right)}, \\
& \beta=\frac{1}{2}(X-Y) e^{-i k\left(\eta-\eta_{i}\right)},
\end{aligned}
$$

the system of Eqs. (22) and (23) become simply

$$
\begin{aligned}
& X^{\prime \prime}+\left(k^{2}-\frac{a^{\prime \prime}}{a}\right) X=0, \\
& Y=\frac{i}{k} X^{\prime} .
\end{aligned}
$$

These differential equations can be solved exactly for pure power-law inflation. Indeed, for $a(\eta) \propto\left(\eta_{1}-\eta\right)^{p}$, Eq. (26) can be written as

$$
\frac{d^{2} X}{d y^{2}}+\left(1-\frac{4 \mu^{2}-1}{4 y^{2}}\right) X=0
$$

which admits the solution

$$
X(y)=y^{1 / 2}\left[c_{1} J_{\mu}(y)+c_{2} Y_{\mu}(y)\right],
$$

where $y=k\left(\eta_{1}-\eta\right), \mu= \pm(1 / 2-p)$, and $J_{\mu}$ and $Y_{\mu}$ are Bessel functions of the first and the second kind.

The functions $X$ and $Y$ should satisfy the initial conditions $|X(\eta=-\infty)|=1$ and $|Y(\eta=-\infty)|=1$, corresponding to $|\alpha(\eta=-\infty)|=1$ and $|\beta(\eta=-\infty)|=0$. This implies that the integration constants should be $c_{1}=\sqrt{\pi / 2} e^{i \delta}$ and $c_{2}= \pm i \sqrt{\pi / 2} e^{i \delta}$, where $\delta$ is an arbitrary real constant. The solution of Eq. (26), which satisfies appropriate initial conditions, is then

$$
X(y)=\sqrt{\frac{\pi y}{2}} H_{1 / 2-p}^{(1,2)}(y) e^{i \delta},
$$

where $H_{1 / 2-p}^{(1,2)}=J_{1 / 2-p} \pm i Y_{1 / 2-p}$ denotes the Hankel functions.

From Eq. (27) is now straightforward to obtain

$$
Y(y)=-i \sqrt{\frac{\pi y}{2}}\left[H_{-1 / 2-p}^{(1,2)}(y)+\frac{p}{y} H_{1 / 2-p}^{(1,2)}(y)\right] e^{i \delta}
$$

which satisfies the initial condition $|Y(\eta=-\infty)|=1$.
At the end of the pure power-law inflationary period, where our numerical integration starts $\left(\eta=\eta_{i}\right)$, the functions $X$ and $Y$ are given by

$$
\begin{aligned}
& X\left(\eta_{i}\right)=\sqrt{\frac{\pi y_{i}}{2}} H_{1 / 2-p}^{(1,2)}\left(y_{i}\right), \\
& Y\left(\eta_{i}\right)=-i \sqrt{\frac{\pi y_{i}}{2}}\left[H_{-1 / 2-p}^{(1,2)}\left(y_{i}\right)+\frac{p}{y_{i}} H_{1 / 2-p}^{(1,2)}\left(y_{i}\right)\right],
\end{aligned}
$$

where $y_{i}=k\left(\eta_{1}-\eta_{i}\right)=-k p a\left(\eta_{i}\right) / a^{\prime}\left(\eta_{i}\right)$ and we have chosen the arbitrary constant $\delta$ to be zero. Equations (32) and (33) will be used as initial conditions for $X$ and $Y$ in our numerical simulations.

\section{NUMERICAL SIMULATIONS}

The numerical simulations begin at the end of the pure power-law inflationary period, $\eta_{i}$, and continue up to the present epoch, $\eta_{0}$. We use a Runge-Kutte method to solve the system of differential equations (16)-(19). Equations (15) and (20) are used as constraint equations to check the accuracy of the numerical solution.

As initial conditions we choose $a\left(\eta_{i}\right)=1$ and $\phi\left(\eta_{i}\right) \equiv$ $\phi_{i}=-0.3 m_{\mathrm{P}}$. Taking into account that, for $\phi \leqslant \phi_{i}$, $\phi^{\prime 2} /\left(2 a^{2}\right)=V(\phi)(p+1) /(2 p-1)$, we obtain

$$
\phi^{\prime}\left(\eta_{i}\right)=\sqrt{\frac{2(p+1)}{2 p-1} V_{0} e^{-\lambda \phi_{i}}},
$$

where $\lambda$ and $V_{0}$ are given by Eqs. (8) and (13), respectively. Since any pre-existing radiation fluid is diluted during inflation, we choose $\rho_{\text {rad }}\left(\eta_{i}\right)=0$. Finally, $a^{\prime}\left(\eta_{i}\right)$ is determined from the constraint (15) to be

$$
a^{\prime}\left(\eta_{i}\right)=\sqrt{3 \pi \frac{p+1}{2 p-1} P_{s}\left(k_{c}\right)} e^{-N_{c}(p+1) / p} t_{\mathrm{P}}^{-1},
$$

where $t_{\mathrm{P}}$ is the Planck time and we have taken into account that $U_{0}\left(e^{\nu \phi_{i}}-1\right)^{n}=V_{0} e^{-\lambda \phi_{i}}$.

Having determined the time evolution of $a^{\prime \prime} / a$ from Eqs. (16)-(19), we then solve Eqs. (26) and (27) (again with a Runge-Kutte method) for different values of $\omega\left(\eta_{0}\right)=k / a\left(\eta_{0}\right)$ and with initial conditions (32) and (33). Finally, we compute $\beta^{2}$ with Eq. (25) and obtain $\Omega_{\mathrm{GW}}\left(\eta_{0}\right)$ from Eq. (21).

The gravitational-wave energy spectrum can be divided in three regions. In the first region, $\omega_{0} \equiv$ $\omega\left(\eta_{0}\right)$ ranges from $\omega_{\min }$ to $\omega_{\mathrm{rad} \rightarrow \text { mat }}$, where $\omega_{\min }=$ $2 \pi c / d_{\text {Hubble }}\left(\eta_{0}\right) \approx 2 \pi H\left(\eta_{0}\right)=1.4 \times 10^{-17} \mathrm{rad} / \mathrm{s}$ is the angular frequency of a gravitational wave with a wavelength equal, today, to the Hubble distance, and $\omega_{\text {rad } \rightarrow \text { mat }}$ is today's value of the angular frequency of a gravitational wave which had a wavelength equal to the Hubble distance at the time when the energy density of radiation became equal to the energy density of matter (corresponding to the transition from a radiation-dominated to a matter-dominated universe). Because of red-shifting, 


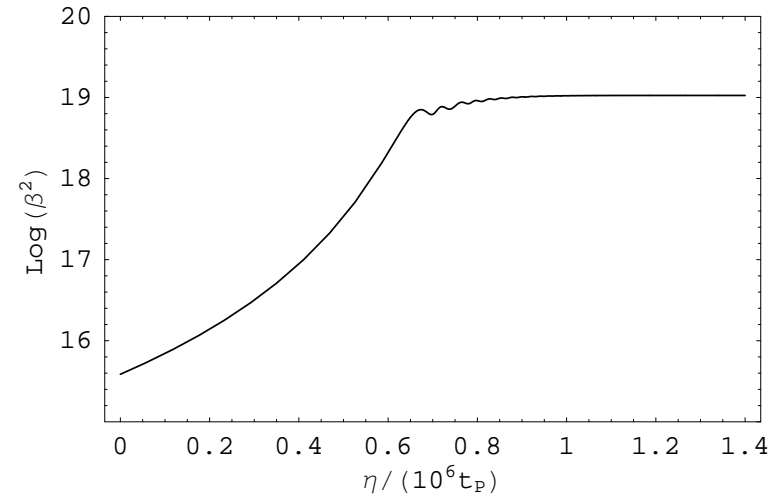

FIG. 2: Time evolution of $\beta^{2}$ during the transition from the inflationary period to the radiation-dominated era, for $\omega_{0}=$ $10^{4} \mathrm{rad} / \mathrm{s}, p=-1.05, \phi_{i}=-0.3 m_{\mathrm{P}}, \Gamma_{\phi}=5.0 \times 10^{-7} m_{\mathrm{P}}$ and $n=2$.

$\omega_{\mathrm{rad} \rightarrow \mathrm{mat}}$ is of the order of $10^{-15} \mathrm{rad} / \mathrm{s}$. In the second (intermediate) region, $\omega_{0}$ ranges from $\omega_{\text {rad } \rightarrow \text { mat }}$ to $\omega_{\phi \rightarrow \mathrm{rad}}$, where $\omega_{\phi \rightarrow \mathrm{rad}}$ is today's value of the angular frequency of a gravitational wave which had a wavelength equal to the Hubble distance at the time when the energy density of the inflaton became equal to the energy density of radiation (marking the end of the transition period between the inflationary and radiation-dominated eras); this frequency is of the order of $10^{7} \mathrm{rad} / \mathrm{s}$. Finally, in the third region, $\omega_{0}$ ranges from $\omega_{\phi \rightarrow \mathrm{rad}}$ to $\omega_{\max }$, where the maximum angular frequency, which is of the order of $10^{10} \mathrm{rad} / \mathrm{s}$, is today's value of the angular frequency of a gravitational wave which had a wavelength equal to the Hubble distance at the end of the inflationary period.

Let us now show, using the method of continuous Bogoliubov coefficients, that in the second (intermediate) region of the spectrum, the relative logarithmic energy density of the gravitational waves, contrarily to the situation in exponential inflation, depends on the frequency of the waves, namely, $\Omega_{\mathrm{GW}} \propto \omega_{0}^{2(p+1)}$.

Taking into account that, according to our numerical simulations, the scale factor at the present time is of the order of $10^{30}$ [for $a\left(\eta_{i}\right)=1$ ], and using Eq. (35) to evaluate $a^{\prime}\left(\eta_{i}\right)$, we obtain

$$
y_{i} \approx-3.5 \times 10^{-10} p \sqrt{\frac{2 p-1}{p+1}} e^{55(p+1) / p} \omega_{0} \mathrm{~s} .
$$

Let us consider values of $p$ in the interval $-1.053 \leqslant p \leqslant$ $-1-\epsilon$. From Eq. (36) follows that $y_{i}<1$ for $\omega_{0} \leqslant 2.2 \times$ $10^{7} \mathrm{rad} / \mathrm{s}$ and $\epsilon=1.9 \times 10^{-4}$, allowing us to expand $X\left(\eta_{i}\right)$ and $Y\left(\eta_{i}\right)$, given by Eqs. (32) and (33), in converging power series. Keeping just the leading term, $X\left(\eta_{i}\right) \propto y_{i}^{p}$ and $Y(\eta) \propto y_{i}^{p-1}$, we obtain that $\beta^{2}\left(\eta_{i}\right) \propto y_{i}^{2(p-1)} \propto$ $\omega_{0}^{2(p-1)}$.

In order to determine the subsequent time evolution of $\beta^{2}$ we have to solve Eq. (26). Let us consider separately three periods: $i$ ) the transition from inflation to the radiation-dominated era $\left.\left(\eta_{i} \leqslant \eta<\eta_{\phi \rightarrow \mathrm{rad}}\right) i i\right)$

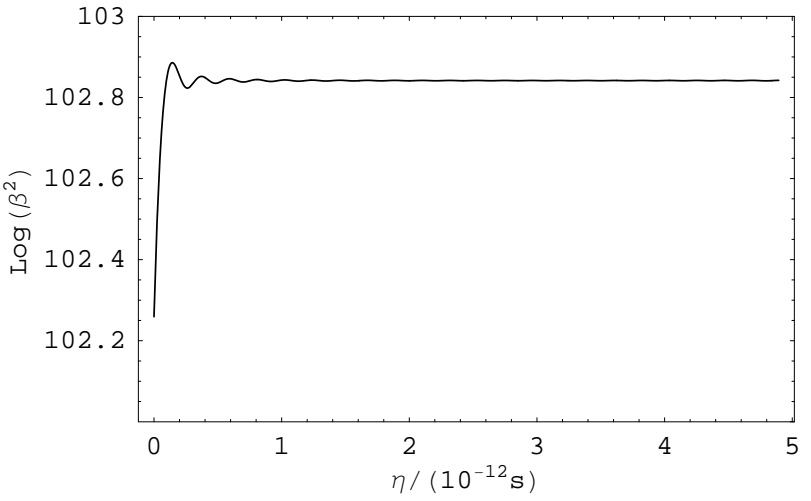

FIG. 3: Time evolution of $\beta^{2}$ during the matter- and dark energy-dominated eras, for $\omega_{0}=5 \times 10^{-17} \mathrm{rad} / \mathrm{s}, p=-1.05$, $\phi_{i}=-0.3 m_{\mathrm{P}}, \Gamma_{\phi}=5.0 \times 10^{-7} m_{\mathrm{P}}$ and $n=2$.

the radiation-dominated era $\left(\eta_{\phi \rightarrow \mathrm{rad}} \leqslant \eta<\eta_{\mathrm{rad} \rightarrow \text { mat }}\right)$ and iii) the matter- and dark energy-dominated eras $\left(\eta_{\mathrm{rad} \rightarrow \text { mat }} \leqslant \eta \leqslant \eta_{0}\right)$.

During the transition from inflation to the radiationdominated era, $a^{\prime \prime} / a$ is a rather complicated function of time and, therefore, Eq. (26) must be solved numerically. In Fig. 2 the time evolution of $\beta^{2}$ is shown for $\omega_{0}=$ $10^{4} \mathrm{rad} / \mathrm{s}, p=-1.05, \phi_{i}=-0.3 m_{\mathrm{P}}, \Gamma_{\phi}=5.0 \times 10^{-7} m_{\mathrm{P}}$ and $n=2$. For other values of $\omega_{0}$, the time evolution of $\beta^{2}$ is the same, provided that $\omega_{0} \leqslant 2.2 \times 10^{7} \mathrm{rad} / \mathrm{s}$. Indeed, for such values of $\omega_{0}, k^{2}=a_{0}^{2} \omega_{0}^{2}<1.4 \times 10^{-12} t_{\mathrm{P}}^{-2}$ is always smaller than $a^{\prime \prime}\left(\eta_{i}\right) / a\left(\eta_{i}\right)$. To see this, we insert $\phi^{\prime}\left(\eta_{i}\right)$ from Eq. (34) into Eq. (16), use the fact that $U_{0}\left(e^{\nu \phi_{i}}-1\right)^{n}=V_{0} e^{-\lambda \phi_{i}}$, with $\lambda$ and $V_{0}$ given by Eqs. (8) and (13), obtaining, for $\epsilon=1.9 \times 10^{-4}$,

$$
\begin{aligned}
\frac{a^{\prime \prime}}{a}\left(\eta_{i}\right) & =3 \pi \frac{p^{2}-1}{p(2 p-1)} P_{s}\left(k_{c}\right) e^{-2 N_{c}(p+1) / p} t_{\mathrm{P}}^{-2} \\
& >2.9 \times 10^{-12} t_{\mathrm{P}}^{-2} .
\end{aligned}
$$

In the first stages of the transition between the inflationary and radiation-dominated eras, $a^{\prime \prime} / a$ increases and the relation $k^{2}<a^{\prime \prime} / a$ continues to hold. Therefore, we can neglect $k^{2}$ in Eq. (26), thus obtaining the same time evolution of $\beta^{2}$ for different values of $\omega_{0}$. To the end of the transition, $a^{\prime \prime} / a$ approaches zero and graviton production ceases, with $\beta^{2}$ becoming constant (see Fig. (2)).

During the radiation-dominated era, $\beta^{2}$ remains constant. Indeed, during this era the scale factor is proportional to the conformal time, implying $a^{\prime \prime} / a=0$. Then Eqs. (26) and (27) admit the solution

$$
\begin{aligned}
& X(\eta)=X\left(\eta_{\phi \rightarrow \mathrm{rad}}\right) \cos q(\eta)-i Y\left(\eta_{\phi \rightarrow \mathrm{rad}}\right) \sin q(\eta), \\
& Y(\eta)=Y\left(\eta_{\phi \rightarrow \mathrm{rad}}\right) \cos q(\eta)-i X\left(\eta_{\phi \rightarrow \mathrm{rad}}\right) \sin q(\eta),
\end{aligned}
$$

where $q(\eta) \equiv k\left(\eta-\eta_{\phi \rightarrow \mathrm{rad}}\right)$. Now, using Eq. (25)), it is straightforward to show that $\beta^{2}(\eta)=\beta^{2}\left(\eta_{\phi \rightarrow \mathrm{rad}}\right)$.

During the matter- and dark energy-dominated eras, $\beta^{2}$ remains constant, if $\omega_{0} \gtrsim 10^{-15} \mathrm{rad} / \mathrm{s}$. Indeed, for such values of $\omega_{0}$, our numerical simulations show that 


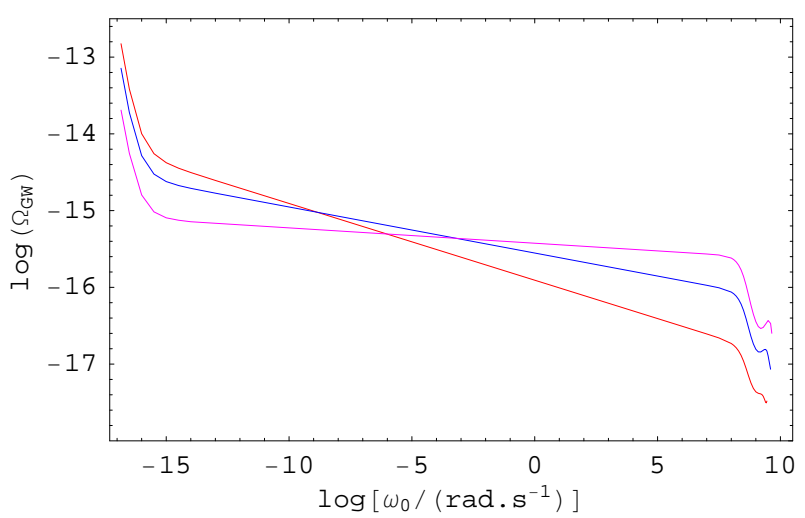

FIG. 4: Gravitational-wave spectra for different values of $p$ : $p=-1.01$ (violet, upper curve at high frequencies), $p=-1.03$ (blue, middle curve) and $p=-1.05$ (red, lower curve). In all spectra we have used $\phi_{i}=-0.3 m_{\mathrm{P}}, \Gamma_{\phi}=5.0 \times 10^{-7} m_{\mathrm{P}}$ and $n=2$.

$k^{2}$ is always greater than $a^{\prime \prime} / a$ and, once again, we can neglect $a^{\prime \prime} / a$ in Eq. (26), obtaining, similarly to the radiation-dominated era, that $\beta^{2}$ remains constant, i.e., $\beta^{2}(\eta)=\beta^{2}\left(\eta_{\mathrm{rad} \rightarrow \text { mat }}\right)$.

Putting everything together, we arrive at the conclusion that, for $10^{-15} \mathrm{rad} / \mathrm{s} \lesssim \omega_{0} \lesssim 10^{7} \mathrm{rad} / \mathrm{s}$ (intermediate region of the gravitational-wave spectrum), gravitons are produced during the transition from the inflationary period to the radiation-dominated era, but not during the radiation-, matter-, and dark energydominated eras, and that this production does not depend on the value of $\omega_{0}$. This, together with the fact that $\beta^{2}\left(\eta_{i}\right) \propto \omega_{0}^{2(p-1)}$, implies that $\beta^{2}\left(\eta_{0}\right) \propto \omega_{0}^{2(p-1)}$. Now, using Eq. (21), we finally obtain that $\Omega_{\mathrm{GW}}\left(\eta_{0}\right) \propto \omega_{0}^{2(p+1)}$, i.e., for $10^{-15} \mathrm{rad} / \mathrm{s} \lesssim \omega_{0} \lesssim 10^{7} \mathrm{rad} / \mathrm{s}$ the relative logarithmic energy density of the gravitational waves depends on the frequency of the waves, in agreement with the results obtained by Sahni using a different method [10]. This contrasts with the results obtained for exponential inflation, where the spectrum in the intermediate region is flat.

Let us point out that for $1.4 \times 10^{-17} \mathrm{rad} / \mathrm{s} \lesssim \omega_{0} \lesssim$ $10^{-15} \mathrm{rad} / \mathrm{s}$, the energy spectrum is steeper than in the intermediate-frequency region. This is due to the fact that, in the beginning of the matter-dominated era, $a^{\prime \prime} / a$ is greater than $k^{2}$ for such range of frequencies, leading to an extra production of gravitons. After a while, $a^{\prime \prime} / a$ approaches zero and graviton production ceases, with $\beta^{2}$ becoming constant (see Fig. 3).

We now produce a few gravitational-wave spectra, coming from our numerical simulations. For each spectrum, we have to specify the values of $p, \phi_{i}, n$ and $\Gamma_{\phi}$. While $p$ is constrained by measurements of the cosmic microwave background and large-scale structure to lie in the interval $-1.053 \leqslant p<-1$, the latter three parameters can be chosen freely. Our choice of $p$ fixes $V_{0}$ and $\lambda$ and, thus, the level of the spectrum.

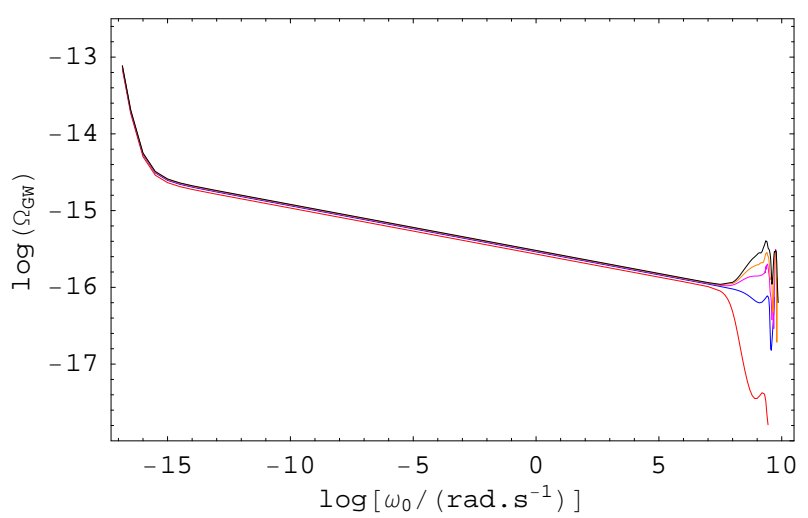

FIG. 5: Gravitational-wave spectra for different values of $n$ : $n=2$ (red, lower curve), $n=4$ (blue, second curve from below), $n=6$ (violet, third curve from below), $n=8$ (orange, second curve from above) and $n=12$ (black, upper curve). In all spectra we have used $p=-1.03, \phi_{i}=-0.3 m_{\mathrm{P}}$ and $\Gamma_{\phi}=5.0 \times 10^{-8} m_{\mathrm{P}}$

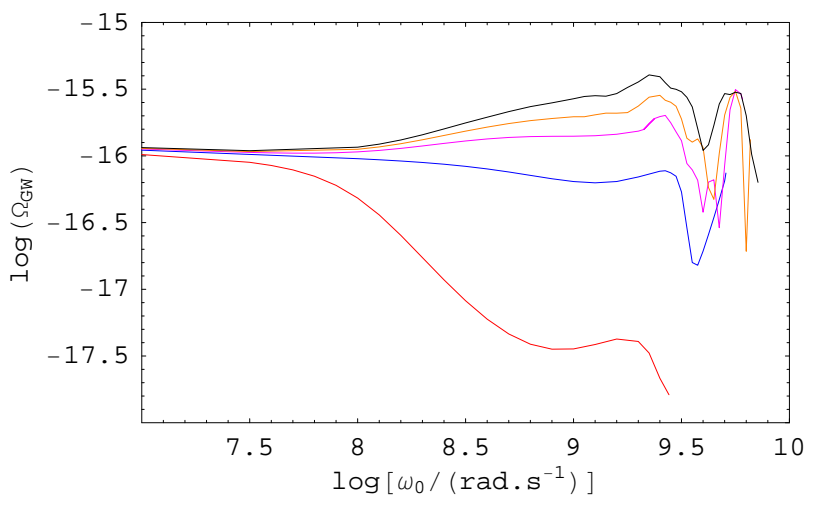

FIG. 6: Zoom of the high-frequency region of the spectra shown in figure 5 .

Gravitational-wave energy spectra, for different values of $p$, are shown in Fig. 4. For intermediate values of the gravitational-wave frequency $\left(10^{-15} \mathrm{rad} / \mathrm{s} \lesssim \omega_{0} \lesssim\right.$ $10^{7} \mathrm{rad} / \mathrm{s}$ ) the spectrum has a slope that depends on the value of $p$. When $p$ approaches -1 , the model becomes closer to exponential inflation and the spectrum closer to flat, as expected. For $\omega_{0} \lesssim 10^{-15} \mathrm{rad} / \mathrm{s}$, the energy spectrum is steeper than in the intermediate-frequency region, as explained above. The behavior in the highfrequency region of the spectra $\left(\omega_{0} \gtrsim 10^{7} \mathrm{rad} / \mathrm{s}\right)$ can be understood as follows. Near its minimum, the potential $V(\phi)$ is proportional to $\phi^{n}$. An homogeneous scalar field, with a potential $V(\phi) \propto \phi^{n}$, oscillating rapidly relatively to the expansion rate of the universe, behaves as a fluid with an equation of state $p=\gamma \rho, \gamma$ depending on $n$ in such a way that the fluid becomes more and more stiff as $n$ increases [27]. Furthermore, the relative logarithmic energy density of the gravitational waves decreases at high-frequencies if inflation is followed by a period 
dominated by a fluid with a dust-like equation of state $(p=0)$, while an increase of the relative energy density is observed at high frequencies if inflation is followed by a stiff matter phase $(p=\rho)$ [26]. Since the spectra in Fig. 4 were obtained for $n=2$, in which case the rapidlyoscillating scalar field behaves like dust, a decrease at high frequencies of $\Omega_{\mathrm{GW}}$ is expected.

The dependence on the parameter $n$ of the relative logarithmic energy density of the gravitational waves at high frequencies is clearly illustrated in Figs. 5 and 6 , where the gravitational-wave energy spectra for different values of $n$ are shown. The part of the spectra corresponding to frequencies between $10^{7} \mathrm{rad} / \mathrm{s}$ and $10^{10} \mathrm{rad} / \mathrm{s}$ does reflect some of the characteristics of the transition between the inflationary and radiation-dominated eras, namely, an increase in the value of the parameter $n$ corresponds to an increase of the relative logarithmic energy density of the gravitational waves produced during the period of thermalization near the minimum of the potential.

Measurements of the cosmic microwave background radiation impose, for angular frequencies corresponding to the present size of the cosmological horizon $\omega_{\text {hor }}=$ $1.4 \times 10^{-17} \mathrm{rad} / \mathrm{s}$, an upper limit on the relative logarithmic energy density of gravitational waves, namely, $\Omega_{\mathrm{GW}}\left(\omega_{\text {hor }}, \eta_{0}\right)<1.4 \times 10^{-10}[1]$. All the spectra presented in this paper satisfy, by far, this condition. In fact, one could even relax the constraints on $p$ and $V_{0}$, discussed in Sec. III and still obtain gravitationalwave energy spectra satisfying the above constraint on $\Omega_{\mathrm{GW}}\left(\omega_{\mathrm{hor}}, \eta_{0}\right)$.

\section{CONCLUSIONS}

In this work, we have investigated the generation of gravitational waves in power-law inflationary models. The parameters of the inflationary potential were constrained using recent measurements from the cosmic microwave background and large-scale structure. We have incorporated reheating in our model through an elementary decay mechanism of the inflationary scalar field into a relativistic radiation fluid. Despite its simplicity, the reheating potential (14) may contain relevant features of more realistic potentials, as was emphasized through an approximate comparison with the potential derived in Ref. [25] from the Salam-Sezgin model.

We have used the method of continuous Bogoliubov coefficients to calculated the gravitational-wave energy spectrum for different values of the parameters of our model. All the spectra we have obtained satisfy the constraint imposed by measurements of the cosmic microwave background radiation at angular frequencies corresponding to the present size of the cosmological horizon. Between $10^{-15} \mathrm{rad} / \mathrm{s}$ and $10^{7} \mathrm{rad} / \mathrm{s}$ the spectrum is not flat, but has a slope that depends on the value of $p$, and so on the value of the parameter $\lambda$ defining the inflationary potential (Fig. 4). When $p$ approaches -1 , the model becomes closer to exponential inflation and the spectrum closer to flat, as expected. The influence of the inflationary regime does extend a long way towards low frequencies and the observation, or non-observation, of such a slope could, in principle, give us information on the type of inflation. On the other hand, the part of the spectrum corresponding to the frequencies between $10^{7} \mathrm{rad} / \mathrm{s}$ and $10^{10} \mathrm{rad} / \mathrm{s}$ does reflect some of the characteristics of the transition between the inflationary and radiation-dominated eras, in particular the behavior during the period of thermalization near the minimum of the potential, as explained at the end of the preceding section, and shown in Figs. 5 and 6 This is why we believe it is important to continue work already done to develop detectors covering the $\mathrm{MHz}$ and $\mathrm{GHz}$ range of frequencies: they might give us information difficult to obtain by any other means. This was one point we wanted to make with our present work, notwithstanding the fact that the model we used was a very simple one.

\section{Acknowledgments}

This work was supported in part by the Fundação para a Ciência e a Tecnologia, Portugal.
[1] B. Allen, Phys. Rev. D 37, 2078 (1988); B. Allen, in Proceedings of the Les Houches School on Astrophysical Sources of Gravitational Waves, Les Houches, France, 1995, edited by J.-A. Marck and J.-P. Lasota (Cambridge University Press, 1997), p. 373.

[2] L. P. Grishchuk, Sov. Phys. JETP 40, 409 (1974).

[3] A. A. Starobinsky, JETP Lett. 30, 682 (1979).

[4] V. A. Rubakov, M. V. Sazhin, and A. V. Veryaskin, Phys. Lett. B 115, 189 (1982).

[5] R. Fabbri and M. D. Pollock, Phys. Lett. B 125, 445 (1983).

[6] L. F. Abbott and M. B. Wise, Nucl. Phys. B 244, 541 (1984).

[7] L. F. Abbott and D. D. Harari, Nucl. Phys. B 264, 487
(1986).

[8] URL http://universe.nasa.gov/program/bbo.html

[9] N. Seto, S. Kawamura, and T. Nakamura, Phys. Rev. Lett. 87, 221103 (2001).

[10] V. Sahni, Phys. Rev. D 42, 453 (1990); T. Souradeep and V. Sahni, Mod. Phys. Lett. A 7, 3541 (1992).

[11] Ph. Bernard, G. Gemme, R. Parodi, and E. Picasso, Rev. Sci. Instrum. 72, 2428 (2001); A. M. Cruise and R. M. J. Ingley, Classical Quantum Gravity 22, S479 (2005).

[12] J. H. Traschen and R. H. Brandenberger, Phys. Rev. D 42, 2491 (1990); L. A. Kofman, A. D. Linde, and A. A. Starobinsky, Phys. Rev. D 56, 3258 (1997).

[13] S. Khlebnikov and I. Tkachev, Phys. Rev. D 56, 653 (1997); R. Easther and E. A. Lim, J. Cosmol. Astropart. 
Phys. 04, 010 (2006).

[14] P. M. Sá and A. B. Henriques, Phys. Rev. D 77, 064002 (2008).

[15] L. Parker, Phys. Rev. 183, 1057 (1969).

[16] R. G. Moorhouse, A. B. Henriques, and L. E. Mendes, Phys. Rev. D 50, 2600 (1994).

[17] L. E. Mendes, A. B. Henriques, and R. G. Moorhouse, Phys. Rev. D 52, 2083 (1995).

[18] F. Lucchin and S. Matarrese, Phys. Rev. D 32, 1316 (1985).

[19] A. H. Guth and S.-Y. Pi, Phys. Rev. Lett. 49, 1110 (1982); S. W. Hawking, Phys. Lett. B 115, 295 (1982); A. A. Starobinsky, Phys. Lett. B 117, 175 (1982); J. M. Bardeen, P. J. Steinhardt, and M. S. Turner, Phys. Rev. D 28, 679 (1983).
[20] T. L. Smith, M. Kamionkowski, and A. Cooray, Phys. Rev. D 73, 023504 (2006).

[21] A. R. Liddle and D. H. Lyth, Phys. Lett. B 291, 391 (1992).

[22] A. Kosowsky and M. S. Turner, Phys. Rev. D 52, R1739 (1995).

[23] D. H. Lyth, Phys. Rev. Lett. 78, 1861 (1997).

[24] A. Salam and E. Sezgin, Phys. Lett B 147, 47 (1984).

[25] L. Anchordoqui, H. Goldberg, S. Nawata, and C. Nuñez,Phys. Rev. D 76, 126005 (2007).

[26] A. B. Henriques, Classical Quantum Gravity 21, 3057 (2004); 24, 6431(E) (2007).

[27] M. S. Turner, Phys. Rev. D 28, 1243 (1983). 